\title{
Prevalence of Domestic Violence among Pregnant Women Attending Primary Health Care Clinics in Gaza
}

\author{
Eslam Farhat Murtaja ${ }^{1}$ and Abdel Aziz Thabet ${ }^{2 \star}$
}

${ }^{1} \mathrm{MPH}$, Community Mental Health, Al Quds University, Palestine

${ }^{2}$ Emeritus Professor of Psychiatry, Al Quds University, School of Public- Consultant Psychiatrist at Child and Family Training and Counseling Center, Gaza, Palestine

\begin{abstract}
Aim: The aim of this study was to explore the prevalence of domestic violence, among pregnant women attending primary health care clinics in Gaza Strip.

Method: Descriptive analytic, cross sectional design. The sample consisted of 400 pregnant women from the five governorates of Gaza Strip. Tools of the study included socio-demographic characteristic questionnaire and Conflict Tactics Scale.

Results: The study showed that psychological assault reported by pregnant women was $6.11 \%$, physical assault was $2.8 \%$, physical injury was $1.3 \%$, and sexual assault was $0.4 \%$. However, psychological assault was significantly higher in women living in camps than women living in cities or villages, women in age group of 36 and above years, uneducated women, uneducated husbands, and having 8 and more children. Moreover, physical assault was significantly more in uneducated women and in women in families with 8 and more. While, physical injury was significantly more in women with uneducated husbands and families with 8 and more children.

Clinical implications: Our study showed that pregnant Palestinian women exposed to physical violence and psychological violence. More specific programs should be established in Gaza to enable women of using new coping strategies with difficulties. Also, training programs including primary health care professional such physicians, nurses, social service experts, and midwives should be provide to enable them of early detection of victims of violence and provide social support to these women.
\end{abstract}

Keywords: Domestic violence; Pregnant women; Gaza strip; Primary health care

\section{Introduction}

Violence is any act or failure to act of a household member against another member in the household for the purpose of causing physical, sexual or psychological abuse, or the threat of physical, sexual or psychological abuse, or generates fear. It also includes the deprivation of basic rights such as shelter, food, drink, clothing, education, freedom of movement and loss of self-determination and self-security [1].

The violence against women is pervasive globally. The findings send a powerful message that violence against women is not a small problem that only occurs in some pockets of society, but rather is a global public health problem of epidemic proportions, requiring urgent action. It is time for the world to take action: a life free of violence is a basic human right, one that every woman, man and child deserves [2]. Domestic violence against women is defined as a pattern of intentional, coercive and violent behavior toward an individual with whom there is or has been an intimate relationship [3]. The United Nation's definition of violence against women includes any act of gender based violence that results in, or is likely to result in, physical, sexual or psychological harm or suffering to women, including threats of such acts, coercion or arbitrary deprivation of liberty, whether occurring in public or private life. Accordingly, physical violence against women encompasses but is not limited to the following [4].

\section{Prevalence of domestic violence}

Overall, 35\% of women worldwide have experienced either physical and/or sexual intimate partner violence or non-partner sexual violence, almost one third (30\%) of all women who have been in a relationship have experienced physical and/or sexual violence by their intimate partner. Thirty-eight percent of women have experienced intimate partner violence. Globally, as many as $38 \%$ of all murders of women are committed by intimate partners. Women who have been physically or sexually abused by their partners report higher rates of a number of important health problems. For example, they are $16 \%$ more likely to have a low-birth-weight baby [2].

Domestic violence among pregnant women is a global health issue. Prevalence of violence among pregnant women in developing countries ranges from $4 \%$ to $29 \%$, but methodological differences among the studies make the actual prevalence rate unclear and not specified for pregnant women only but all women. The World Health Organization defines domestic violence as the range of sexually, psychologically and physically coercive acts used against young and adolescent women by current or former male intimate partners [5]. Traumatic stress is thought to be the main mechanism that explains why intimate partner violence may cause subsequent depression and suicide attempts, and exposures to traumatic events can lead to stress, fear and isolation, which, in turn, may lead to depression and suicidal behavior [2]. In United Kingdom, domestic violence is reported in every one out of four women, while domestic violence against women is found in the form of physical, verbal or psychological abuse in Pakistan [5].

*Corresponding author: Thabet AA, Emeritus Professor of Psychiatry, Al Quds University, School of Public- Consultant Psychiatrist at Child and Family Training and Counseling Center, Gaza, Palestine, Tel: + 00970599604400; E-mail: abdelazizt@hotmail.com

Received: June 19, 2017; Accepted: June 22, 2017; Published: June 29, 2017

Citation: Murtaja EF, Thabet AA (2017) Prevalence of Domestic Violence among Pregnant Women Attending Primary Health Care Clinics in Gaza. Clin Exp Psychol 3: 156. doi: 10.4172/2471-2701.1000156

Copyright: (C) 2017 Murtaja EF, et al. This is an open-access article distributed under the terms of the Creative Commons Attribution License, which permits unrestricted use, distribution, and reproduction in any medium, provided the original author and source are credited. 
A survey by WHO [6] included 10 countries and 24,000 women showed that domestic violence against pregnant women was in the form of physical violence from their partners $(23-49 \%)$ and sexual violence (10-50\%). In Egypt, one third of women are physically abused by their husbands, and most victims suffer silently and don't seek help to prevent or stop the violence because they think it is part of life or they are embarrassed by the abuse [7]. In Jordan, one in four women was at risk of physical violence committed by husbands, fathers, and brothers [8]. According to Palestinian Central Bureau of Statistics [1], 37.0\% of women who ever been married were exposed to one form of violence by their husbands in the past 12 months; $29.9 \%$ in the West Bank compared to $51.0 \%$ in Gaza Strip. The rate of exposure to psychological was $58.6 \%$, and $55.1 \%$ were exposed to economical violence, $54.8 \%$ were exposed to social violence, $23.5 \%$ were exposed to physical violence and $11.8 \%$ were exposed to sexual violence. In Palestine, Thabet et al. [9] in study of 622 women in Gaza Strip reported that psychological assault was $56.91 \%$, physical assault $37.3 \%$, physical injury $12.06 \%$, and sexual assault was $7.14 \%$. The study showed that domestic violence was significantly higher in women living in villages than in cities or camps.

\section{Domestic violence during pregnancy}

During pregnancy a woman may begin to be abused or longstanding abuse may change in severity, which may have negative health affects to the mother and fetus. Pregnancy can also lead to a hiatus of domestic violence when the abuser does not want to harm the unborn child. The risk of domestic violence for women who have been pregnant is greatest immediately after childbirth [10].

Taft and Watson [11] reported that women exposed to violence from their partners have many more unwanted pregnancies, abortions, and pregnancy-related complications. Akyuz et al. [12], showed that Turkish women who experienced more violence did not plan their most recent pregnancies and, therefore, terminated those pregnancies by means of abortion. Hussain and Khan [13] reported that unwanted pregnancies among women experiencing violence have a higher rate being terminated through induced abortion. Furthermore, these unwanted pregnancies can lead to unsafe abortions in countries where abortion is illegal. This situation causes many risks of serious health complications and even death for women exposed to violence. In addition, physical and sexual violence continues during pregnancy for many women if the pregnancy continues. This may affect both the health of the women and the baby [12]. This study aimed to explore the prevalence rate of domestic violence among pregnant women attending primary health care clinics in Gaza Strip.

\section{Methods}

\section{Participants}

The suspected deliveries are around 60,000 per year in Gaza Strip, and by using Epi-info program, the sample size equals 382 at confidence interval 95\%, and the researcher increased the number of participants to 400 pregnant women to cover for possible nonresponse.

\section{Measures}

\section{Sociodemographic sheet}

It included age, educational level for women and their husbands, place of residence, type of housing, family type, number of family members, and monthly income. Revised Conflict Tactics Scale [14]. Conflict Tactics Scale [14] which included subscales measuring physical, sexual, and psychological, and injury due to domestic violence. A previous version of the CTS was used for a study on domestic violence in the Palestinian territories 16 [15]. This self-report instrument measures the frequency of physical abuse, verbal violence, sexual violence, reasoning tactics, and injury within an intimate relationship. Participants were asked to report the frequency with which they performed the various behavioral conflict resolution tactics in the previous year. Specifically, 12 items from the Physical Assault subscale, 8 items from the Psychological Aggression subscale, 3 items from the Sexual Coercion subscale, and 6 items from the Injury subscale were used to measure intimate partner physical assault, psychological abuse, and sexual coercion. Participants were asked to report on the frequency of each of the abusive acts occurring in a partner conflict situation over the past year using a 7-point scale, with the following response options: $0=$ never, $1=$ once, $2=$ twice, $3=3$ to 5 times, $4=6$ to 10 times, $5=11$ to 20 times, and $6=$ more than 20 times, $7=$ happened before one year. The total scores for each subscale were calculated by adding up the midpoints of the response categories for each item (e.g., the midpoint for the 6 to 10 times response was 8). Thabet et al. [9] showed that the Cronbach's alpha coefficient was 0.92 for the Physical Assault subscale, 0.88 for the Psychological Aggression subscale, 0.89 for the Sexual Coercion subscale, and 0.88 for the Injury subscale. In this study, the Cronbach's alpha coefficient was 0.89 for the Physical Assault subscale, 0.91 for the Psychological Aggression subscale, 0.88 for the Injury subscale, and 75 for the Sexual Coercion subscale.

\section{Study procedure}

Participants were selected from both governmental and UNRWA health centers in Gaza Strip during their visit to antenatal clinics for follow up. Participants were selected by using systematic sampling method and every third woman was included in the study, taking in consideration that the first one was selected randomly. The data were collected using systematic sampling method from pregnant women during their follow up in antenatal clinics in North Gaza, Gaza, Middle, Khan Younis, and Rafah, both governmental and UNRWA health centers. The clinics were selected randomly from each governorate. To assure equal chance of participation in the study for pregnant women, the researcher collected data at the beginning of the month (day 1, 2, 3), middle of the month (day 14, 15, 16), and at end of the month (day 27, 28, 29). An official approval to conduct the study was obtained from Helsinki Committee, UNRWA health department, and Ministry of Health. In addition, informed written consent from each woman was obtained, furthermore, explanation for women who agreed to participate in the study including the purpose of the study, confidentiality information and some instructions, right to withdraw or refuse to participate in the study. The study collection was carried out during the period from December 2015 to October 2016.

\section{Statistical analysis}

Statistical analysis was carried out using SPSS program version 20. Continuous variables were presented as means and standard deviations, categorical variables were expressed as frequencies and percentage. In addition, independent $t$ test to compare mean of domestic violence and type of clinics was performed. One way ANOVA, and Tukey significant differences tests were used. Another linear regression analysis of each subscales of the domestic violence (physical, psychological, and injury) were entered as dependent variables and other socioeconomic variables as independent variables (age, education of women and husbands, place of residence, family member, and family monthly income). $\mathrm{P}$ value $\leq$ 0.05 was considered statistically significant.

\section{Results}

\section{Socio demographic characteristic for study samples}

According to age, $37.3 \%$ of the sample their age less than 25 years, 
$48.5 \% 25$ to less than 35 years, while $14.3 \%$ more than 35 years. Regard qualification $1.3 \%$ of the sample did not learned, 5.8\% Primary, $11.8 \%$ Preparatory, 37.3\% Secondary, 13\% Diploma, 28.8\% Bachelor, 1.5\% higher Diploma, while 3.0\% have high study qualification. Regard clinic type, $60.0 \%$ of the sample follow at Government clinic, while $40.0 \%$ follow at UNRWA clinic. Regard monthly income $77.3 \%$ of the sample their monthly income less than $\$ 450,12.0 \%$ from $\$ 451-\$ 600,7.0 \%$ from $\$ 601-\$ 800,3.3 \%$ from $\$ 801-\$ 1050$, and while 0.5 had monthly income more than $\$ 1051$ (Table 1).

\section{Means, standard deviation, and percentage of domestic violence}

The study showed that psychological assault reported by pregnant women was $6.11 \%$, physical assault $2.8 \%$, physical injury was $1.3 \%$, and sexual assault was $0.4 \%$ (Table 2).

\begin{tabular}{|c|c|c|}
\hline Item & $\%$ & $N$ \\
\hline \multicolumn{3}{|l|}{ Age } \\
\hline Less than 25 years & 37.3 & 149 \\
\hline 26 to less than 35 years & 48.5 & 194 \\
\hline more than 36 years & 14.3 & 57 \\
\hline \multicolumn{3}{|l|}{ Education of women } \\
\hline Illiterate & 1.3 & 5 \\
\hline Primary & 5.8 & 23 \\
\hline Preparatory & 11.8 & 47 \\
\hline Secondary & 37.3 & 149 \\
\hline Diploma & 13 & 52 \\
\hline University & 28.8 & 115 \\
\hline Higher Diploma & 1.5 & 6 \\
\hline Postgraduate study & 0.8 & 3 \\
\hline \multicolumn{3}{|c|}{ Educational qualification for the husband } \\
\hline Not educated & 2.8 & 11 \\
\hline Primary & 6.5 & 26 \\
\hline Preparatory & 14 & 56 \\
\hline Secondary & 35.3 & 141 \\
\hline Diploma & 10.5 & 42 \\
\hline University & 26 & 104 \\
\hline Higher Diploma & 2 & 8 \\
\hline Postgraduate study & 3 & 12 \\
\hline \multicolumn{3}{|l|}{ Place of residence } \\
\hline City & 59.3 & 237 \\
\hline Camp & 30.3 & 121 \\
\hline Village & 7.3 & 29 \\
\hline Other & 3.3 & 13 \\
\hline \multicolumn{3}{|l|}{ Clinic type } \\
\hline Government & 60 & 240 \\
\hline UNRWA & 40 & 160 \\
\hline \multicolumn{3}{|l|}{ Number of family members } \\
\hline Less than 4 & 36.5 & 146 \\
\hline $5-7$ & 34.5 & 138 \\
\hline 8 and above & 29 & 116 \\
\hline \multicolumn{3}{|l|}{ Monthly income } \\
\hline Less than $\$ 450$ & 77.3 & 309 \\
\hline$\$ 451-\$ 600$ & 12 & 48 \\
\hline$\$ 601-\$ 800$ & 7 & 28 \\
\hline$\$ 801-\$ 1050$ & 3.3 & 13 \\
\hline More than $\$ 1051$ & 0.5 & 2 \\
\hline
\end{tabular}

Table 1: Sociodemographic characteristics of the study sample $(N=400)$.

\begin{tabular}{|c|c|c|c|}
\hline Items & Mean & SD & $\%$ \\
\hline Psychological assault & 13.69 & 14.02 & 6.11 \\
\hline Physical assault & 9.42 & 15.16 & 2.80 \\
\hline Physical injury & 2.19 & 5.30 & 1.30 \\
\hline Sexual assault & 0.36 & 1.67 & 0.42 \\
\hline
\end{tabular}

Table 2: Means, standard deviation, and percentage of domestic violence in pregnant women $(\mathrm{N}=400)$

\section{Domestic violence and socio demographic variables}

In order to investigate the differences in violence and socioeconomic variables such as type of clinics, age, type of residence, education, and monthly family income, an independent $t$ and One-way ANOVA tests were performed in which each of the demographic variable was entered as independent variables and means of violence including subscales as dependent variable. No significant differences in all types of domestic violence and type of clinic (governmental/UNRWA) and age of women as it was recorded in three categories (less than 25 years, 26-35 years, and 36 and above years). Post hoc test using Tukey test revealed that psychological assault was significantly higher in women living in camps than women living in cities or villages, women in age group of 36 and above years $(\mathrm{F}(2,399)=4.64, \mathrm{p}=0.01)$, uneducated women $(\mathrm{F}(7$, $399)=4.73, \mathrm{p}=0.001)$, uneducated husbands $(\mathrm{F}(7,399)=03, \mathrm{p}=0.004)$, and having 8 and more children $(\mathrm{F}(7,399)=13.59, \mathrm{p}=0.001)$. Post hoc test using Tukey test showed that physical assault was significantly more in uneducated women $(\mathrm{F}(7,399)=3.41, \mathrm{p}=0.001)$ and in women in families with 8 and more $(\mathrm{F}(2,399)=5.91, \mathrm{p}=0.003)$. While, physical injury was not no significantly affected by monthly income, age, husbands' education, and place of residence. Post hoc test using Tukey test showed that physical injury was significantly more in women with uneducated husbands $(\mathrm{F}(7,399)=2.17, \mathrm{p}=0.03)$ and families with 8 and more children $(\mathrm{F}(2,399)=5.91, \mathrm{p}=0.003)$. While, physical injury was non-significant according to women age, education, place of residence, and monthly income.

\section{Prediction of psychological assault by sociodemographic variables}

In a Linear regression model, each domestic violence mean subscales scores (physical, psychological, injury, and sexual) were entered as an dependent variables and society demographic variables such as age, education of women, husbands' education, monthly income, and work as independent variables. Psychological assault was predicted by women education in which uneducated women exposed more to psychological assault than women with higher education $(\beta=0.20, t(400), p<0.001)$, and women with 8 and more children were significantly predicted psychological assault $(\beta=0.19, \mathrm{t}(400), \mathrm{p}<0.001)$, $\mathrm{R} 2=0.09, \mathrm{~F}(1,400)=21.3, \mathrm{p}<0.001$ (Table 3 ).

\section{Prediction of physical assault by sociodemographic variables}

Physical assault was predicted by women education in which uneducated women exposed more to psychological assault than women with higher education $(\beta=0.14, \mathrm{t}(400), \mathrm{p}<0.001)$, and women with 8 and more children were significantly predicted psychological assault $(\beta=0.18, \mathrm{t}(400), \mathrm{p}<0.001), \mathrm{R} 2=.06, \mathrm{~F}(1,400)=13.35, \mathrm{p}<0.001($ Table $4)$.

\section{Prediction of physical injury by sociodemographic variables}

Psychological assault was predicted by women education in which uneducated women exposed more to psychological assault than women with higher education $(\beta=0.11, \mathrm{t}(400), \mathrm{p}<0.001)$, and women with 8 and more children were significantly predicted psychological assault $(\beta=0.14, \mathrm{t}(400), \mathrm{p}<0.001), \mathrm{R} 2=.03, \mathrm{~F}(1,400)=7.96, \mathrm{p}<0.001$ (Table 5). 


\begin{tabular}{|c|c|c|c|c|c|c|c|}
\hline & \multicolumn{2}{|c|}{ Unstandardized Coefficients } & \multirow{2}{*}{$\begin{array}{c}\text { Standardized Coefficients } \\
\text { Beta }\end{array}$} & \multirow{2}{*}{$\mathbf{t}$} & \multirow{2}{*}{$\mathbf{p}$} & \multicolumn{2}{|c|}{$95.0 \%$ Confidence Interval for B } \\
\hline & B & Std. Error & & & & Lower Bound & Upper Bound \\
\hline (Constant) & 16.76 & 3.23 & & 5.18 & 0.000 & 10.40 & 23.12 \\
\hline Women education & -2.13 & 0.52 & 0.20 & 4.09 & 0.0001 & 3.16 & 1.11 \\
\hline Number of family members & 3.40 & 0.852 & 0.19 & 3.99 & 0.0001 & 1.732 & 5.08 \\
\hline
\end{tabular}

Table 3: Linear regression model of psychological assault with sociodemographic variables.

\begin{tabular}{|c|c|c|c|c|c|c|c|}
\hline & \multicolumn{2}{|c|}{$\begin{array}{l}\text { Unstandardized } \\
\text { Coefficients }\end{array}$} & \multirow{2}{*}{$\begin{array}{c}\text { Standardized } \\
\text { Coefficients } \\
\text { Beta }\end{array}$} & \multirow{2}{*}{$\mathbf{t}$} & \multirow{2}{*}{$\mathbf{p}$} & \multicolumn{2}{|c|}{ 95\% Confidence Interval for B } \\
\hline & B & Std. Error & & & & $\begin{array}{l}\text { Lower } \\
\text { Bound }\end{array}$ & Upper Bound \\
\hline (Constant) & 10.30 & 3.56 & & 2.89 & 0.00 & 3.30 & 17.30 \\
\hline Women education & $-1.62-$ & 0.57 & 0.14 & $-2.82-$ & 0.01 & $-2.75-$ & -0.49 \\
\hline Number of family members & 3.34 & 0.94 & 0.18 & 3.56 & 0.001 & 1.49 & 5.18 \\
\hline
\end{tabular}

Table 4: Linear regression model of physical assault with sociodemographic variables.

\section{Discussion}

Violence against women is increasing global problems which require actions and rules to alleviate its psychosocial consequences. This study assessed the prevalence rate of domestic violence among pregnant women in Gaza Strip. The study showed that psychological assault reported by pregnant women was $6.11 \%$, physical assault was $2.8 \%$, physical injury was $1.3 \%$, and sexual assault was $0.4 \%$. Our study results were inconsistent with study conducted by Cardoza [16] aimed to explore partner violence during pregnancy in Nicaragua. Prevalence of emotional, physical and sexual partner abuse during pregnancy was $32.4 \%, 13.4 \%$ and $6.7 \%$ respectively. Seventeen percent of the victims suffered all three types of violence and in two thirds the abuse was severe and repeated. Half of the abused women had experienced punches and kicks directed to the abdomen; however, only $14 \%$ had sought health care and very few had disclosed the abuse or contacted police or authorities. Adolescent mothers, unwanted pregnancy and late registration for antenatal care or no check-ups were more likely among victims.

Our study was inconsistent with study conducted by Jayasuriya [17] of prevalence estimates for IPV, identify possible risk/protective factors, and describe the care-seeking behavior of abused women Sri Lanka. Findings show that lifetime prevalence of physical violence $(34 \%)$, controlling behavior (30\%) and emotional abuse (19\%) was high and the prevalence of sexual violence was low (5\%). Our explanation to this differences in rate of violence could be due to the fact that both cultures are different in many ways, for Palestinian women, they were affecting war long standing stress and trauma including 3 wars in the last five years and families are considering the mothers are the protecting figure in the family because they are caring for the children during incursions and bombardment, so society considered violence against women is intolerable and especially for pregnant women. The role of extended families also play major role in preventing the violence against the pregnant women. Moreover, most of the Palestinians are Muslims and such society beating and violence against pregnant women is not tolerated.

Our study results of prevalence of domestic violence is much lower than study carried out by Palestinian central of bureau of statistics (PCBS) [1], in which $37.0 \%$ of women who ever been married were exposed to one form of violence by their husbands in the past 12 months; $29.9 \%$ in the West Bank compared to $51.0 \%$ in Gaza Strip. The rate of those who were exposed to psychological violence "at least for one time" among those women out of violated women was $58.6 \%$.
$55.1 \%$ were exposed to economical violence, $54.8 \%$ were exposed to social violence, $23.5 \%$ were exposed to physical violence and $11.8 \%$ were exposed to sexual violence [1]. Our study findings were lower the rate of domestic violence in study women in community sample in the Gaza Strip [9] and Ludermir et al. [18] who found that psychological assault was among the most common forms of violence against women. Furthermore, the results indicated that sexual assault was the lowest form of violence and such findings were consistent with other studies $[17,19]$. Our study showed no significant differences in all types of domestic violence and type of clinic (governmental/ UNRWA) and age of women as it was recorded in three categories (less than 25 years, 26-35 years, and 36 and above years). However, psychological assault was significantly higher in women living in camps than women living in cities or villages, women in age group of 36 and above years, uneducated women, uneducated husbands, and having 8 and more children. Moreover, physical assault was significantly more in uneducated women and in women in families with 8 and more. While, physical injury was significantly more in women with uneducated husbands and families with 8 and more children. Others found other risk factors in women during pregnancy, Naved and Persson [20] aimed to examined factors associated with physical spousal abuse of women during Pregnancy in Bangladesh. The results showed that, Urban and rural women whose mother or mother-in-law had experienced physical spousal abuse had increased odds of experiencing abuse during pregnancy, increased spousal communication was negatively associated with the outcome in both settings. Among urban women, being older than 19, having a husband with more than 10 years of education and being from certain higher income quartiles were negatively associated with abuse, living in a community highly concerned about crime was positively associated with abuse. Also, Hammoury et al. [21], studied domestic violence against pregnant Palestinian refugee women residing in Lebanon and currently using the United Nation Relief and Work Agency's (UNRWA) primary healthcare services. The study result show that, domestic violence was significantly associated with education, gestational age, fear of husband or someone else in the house, and unintended pregnancy. Domestic violence against women in Lebanon was associated with educational level, gestational age, fear of husband or someone else in the house, and unintended pregnancy. Also Assaf and Chaban [22], studied domestic violence against single, nevermarried women in the Occupied Palestinian Territory revealed that decision-making power and age were significant predictors of physical and psychological abuse. Relation to household head, refugee status, education, employment, and locality were not significant; region was only significant for psychological abuse. Furthermore, Zareen et al. 


\begin{tabular}{|c|c|c|c|c|c|c|c|}
\hline & \multicolumn{2}{|c|}{$\begin{array}{l}\text { Unstandardized } \\
\text { Coefficients }\end{array}$} & \multirow{2}{*}{$\begin{array}{c}\text { Standardized } \\
\text { Coefficients } \\
\text { Beta }\end{array}$} & \multirow[t]{2}{*}{$\mathbf{t}$} & \multirow[t]{2}{*}{$\mathbf{p}$} & \multicolumn{2}{|c|}{$95.0 \%$ Confidence Interval for B } \\
\hline & B & Std. Error & & & & Lower Bound & Upper Bound \\
\hline (Constant) & 2.46 & 1.26 & & 1.95 & 0.05 & 0.02 & 4.94 \\
\hline Women education & 0.91 & 0.33 & 0.14 & 2.73 & 0.01 & 0.25 & 1.56 \\
\hline Number of family members & 0.45 & 0.20 & 0.11 & 2.20 & 0.03 & 0.85 & 0.05 \\
\hline
\end{tabular}

Table 5: Linear regression model of physical injury with sociodemographic variables.

[5], in a study aimed to determine the causes and type of domestic violence endured by pregnant women and their effect on pregnancy, in terms of maternal and neonatal outcome. The study showed that the e factors associated with domestic violence included addiction, allegedly aggressive nature of husband, and unemployment of husband. In similar study done by Clark et al. [23] aimed to clarify association between exposure to political violence and intimate-partner violence in the occupied Palestinian territory. The study showed that exposure to political violence was characterized as the husband's direct exposure, his indirect exposure via his family's experiences, and economic effects of exposure on the household. The study finding economic effects of exposure were associated with increased odds of intimate-partner violence in the Gaza Strip only [24,25].

\section{Clinical Implications}

Our study showed that pregnant Palestinian women exposed to physical violence and psychological violence. So there is a great need for more programs for women victims of domestic violence in Palestinian society are needed with well trained professionals in the field of psychological support and therapy. More specific programs should be established in Gaza to enable women of using new coping strategies with difficulties. Also, training programs including primary health care professional such physicians, nurses, social service experts, and midwives should be provide to enable them of early detection of victims of violence and provide social support to these women. Also to provide legal protection for women and fight violence against them, reinforce a secure social culture in fighting all forms of violence against women through enforcing it in the school curriculum, to reinforce the role of religious discourse and direct it towards violence against women, to establish a culture of dialogue and respect of the other in the Palestinian family through an enhancement of the concept of social roles from a gender perspective. Also, we have to establish support psychological department specialized for pregnant women in each maternal clinic. To Encourage positive and responsible reporting and discussion of domestic violence by the media and assigning to the Palestinian media the responsibility for removing the attitudinal barriers and changing behavior and attitudes towards this group.

\section{Conclusion}

Palestinian women, like many other women's in the regions, sustain both direct and indirect violence. Women are subjected to different types of violence during pregnancy. Generally, this result clearly gives us evidence that there is strong relation between violence against women during pregnancy and socio-demographic factors. Mostly sociodemographic conditions appear as prominent risk factors of violence during pregnancy in different contexts and cultures. It is important to understand the risks of violence they may face the pregnant women in the household. This study has found that age, education of pregnant women, educational qualification for the husband, place of residence and family members and decision-making power were significantly associated with domestic violence abuse in the household.

\section{References}

1. Palestinian Central Bureau of Statistics (2012) Violence survey in the Palestinian society. Palestine National Authority, Ramallah, Palestine. pp: 1-41.

2. World Health Organization (2013) Global and regional estimates of violence against women.

3. Leung TW, Leung WC, Chan PL (2002) A comparison of the prevalence of domestic violence between patients seeking termination of pregnancy and other general gynecology patients. Int J Obstet Gynaecol 77: 47-54.

4. Almosaed M (2004) Violence against women: A cross-cultural perspective. Journal of Muslim Affairs 24: 67-88.

5. Zareen N, Majid N, Naqvi S, Saboohi S, Fatima H (2009) Effect of domestic violence on pregnancy outcome. Journal of the College of Physicians and Surgeons Pakistan 19: 291-296.

6. World Health Organization (2005) Country cooperation strategy for and the occupied Palestinian territory. WHO Regional Office for the Eastern Mediterranean.

7. Monazea, Abdel Khalek (2010) Domestic violence high in Egypt, affecting women's reproductive health. Population Reference Bureau.

8. Haddad L, Shotar A, Younger J, Alzyoud S, Bouhaidar C (2011) Screening for domestic violence in Jordan: Validation of an Arabic version of a domestic violence against women questionnaire. Int J Women's Health 9: 79-86.

9. Thabet AA, Tawahina A, Victoria V, Panos Vostanis P (2015) PTSD, Depression, and anxiety among Palestinian women victims of domestic violence in the Gaza Strip. British Journal of Education, Society \& Behavioural Science.

10. Sen $P$ (2000) Enhancing women's choices in responding to domestic violence in Calcutta: A comparison of employment and education. The European Journal of Development Research 11: 65-86.

11. Taft A, Watson L (2007) Termination of pregnancy: Associations with partner violence and other factors in a national cohort of young Australian women. Australian and New Zealand Journal of Public Health 31: 135-142.

12. Akyüz A, Yavan T, Şahiner G, Kiliç A (2012) Domestic violence and woman's reproductive health: A review of the literature. Aggression and Violent Behaviour 17: $514-518$

13. Hussain R, Khan A (2008) Women's perceptions and experiences of sexual violence in marital relationships and its effect on reproductive health. Health Care for Women International 29: 468-483.

14. Straus MA, Hamby SL, Boney-McCoy S, Sugarman DB (1996) The revised conflict tactics scales (CTS-2). Journal of Family Issues 17: 283-316.

15. Haj-Yahia M (2000) Wife abuse and battering in the sociocultural context of Arab society. Family Process 39: 237-255

16. Cardoza E (2005) Partner violence during pregnancy psychosocial factors and child outcomes in Nicaragua. Umea University, Sweden.

17. Jayasuriya V, Wijewardena K, Axemo $P$ (2011) Intimate partner violence against women in the capital province of Sri Lanka: Prevalence, risk factors, and help seeking. Violence against Women 17: 1086-1102.

18. Ludermir A, Valongueiro $S$, Araújo (2014) Common mental disorders and intimate partner violence in pregnancy. Federal University of Pernambuco, Recife, PE, Brazil.

19. Machado M, Monteiro J, Haas V, Abrão A, Sponholz F (2015) Intimate partner violence and anxiety disorders in pregnancy. Rev Latino-Am Enfermagem 23 855-864.

20. Naved R, Persson L (2008) Factors associated with physical spousal abuse of women during pregnancy in Bangladesh. International Family Planning Perspectives. 
Citation: Murtaja EF, Thabet AA (2017) Prevalence of Domestic Violence among Pregnant Women Attending Primary Health Care Clinics in Gaza. Clin Exp Psychol 3: 156. doi: 10.4172/2471-2701.1000156

Page 6 of 6

21. Hammoury N, Khawaja M, Mahfoud Z, Afifi RA, Madi H (2009) Domestic Violence Against Women During Pregnancy. J Women Health 18: 337-345

22. Assaf SH, Chaban S (2013) Domestic violence against single, never- married women in the occupied Palestinian territory. Violence against Women 19: 422 441.

23. Clark CJ, Everson-Rose SA, Suglia SF, Btoush R, Alonso A, et al. (2010) Association between exposure to political violence and intimate- partner violence in the occupied Palestinian territory: A cross-sectional study. The Lancet 375: 310-316.

24. Karmaliani R, Asad N, Bann CM, Moss N, Mcclure EM, et al. (2009) Prevalence of anxiety, depression and associated factors among pregnant women of Hyderabad. Pakistan International Journal of Social Psychiatry 55: 414-424.

25. Marcus SM, Flynn HA, Blow FC, Barry KL (2003) Depressive symptoms among pregnant women screened in obstetrics settings. J Women's Health 12: 373-380. 